\title{
BISTUA
}

Revista de la Facultad de Ciencias Básicas

ISSN: 0120-4211 - ISSN-e: 2711-3027

\section{Incremento del desempeño del sistema de perforación y voladura en la explotación minera el Lucero.}

\author{
Increased performance of the drilling and blasting system at the El Lucero mining operation
}

\author{
Jolman Montaño ${ }^{a}$; José Rincón ${ }^{a}$; Wilson Vargas ${ }^{a}$; Héctor Alvarado ${ }^{a}$ \\ ${ }^{a}$ Escuela de Ingenieros Militares. Bogotá, Colombia.
}

Correspondencia: jfrinconf@gmail.com,Jorge.fonsecab@esing.edu.co

Recibido: Mayo17, 2021. Aceptado: Junio 9, 2021. Publicado: Junio 15, 2021

\begin{abstract}
Resumen
El presente artículo expone el trabajo realizado en la investigación Incremento del desempeño del sistema de perforación y voladura en la explotación minera el Lucero, asociando la eficiencia de estos sistemas a la productividad y rentabilidad. El diseño técnico de la malla de perforación y voladura, involucra las características geológicas y geomecánicas del macizo rocoso, permitiendo alcanzar objetivos concretos de productividad, optimización e incremento de eficiencia de la mina subterránea de carbón el Lucero ubicada en la vereda de Peñas de Cajón del municipio de Sutatausa Cundinamarca.

Un hecho a evitar en las explotaciones mineras en socavón es la perforación deficiente, esta anomalía se mitiga con el conocimiento de las características geológicas del macizo rocoso. Al realizar la integración de los sistemas de perforación y voladura al área productiva se favorece la efectividad del método productivo.
\end{abstract}

Palabras clave: sistema de perforación; voladura y macizo rocoso.

\begin{abstract}
This article presents the work carried out in the investigation Increasing the performance of the drilling and blasting system in the El Lucero mining exploitation, associating the efficiency of these systems with productivity and profitability. The technical design of the drilling and blasting involve geological and geomechanical characteristics of the rocky massif, makes it possible to achieve specific objectives of productivity, optimization and increased efficiency of the El Lucero underground coal mine located in the Peñas de Cajón of the municipality of Sutatausa Cundinamarca.

A fact to avoid in mining operations in a sinkhole is poor drilling, this anomaly is mitigated with the knowledge of the geological characteristics of the rock massif. By integrating the drilling and blasting systems into the production area, the effectiveness of the production method is favored.
\end{abstract}

Keywords: drilling system; blasting and rock mass

\section{Introducción}

La constante preocupación por generar procesos productivos eficientes y que cumplan con los requerimientos de la norma nacional ha impulsado el desarrollo de la investigación "Incremento del desempeño del sistema de perforación y diseño de voladura en la explotación minera el Lucero.”, para tal fin se estableció el siguiente interrogante de investigación ¿Cómo optimizar Incremento del desempeño del desempeño del sistema de perforación y diseño de voladura en la explotación minera el Lucero? teniendo en cuenta este interrogante se analizaron las variables en las que se deben cimentar el diseño de un sistema de perforación y voladura. Al realizar estos procedimientos descritos hasta aquí es fundamental evaluar la funcionalidad y efectividad de los procesos de explotación minera.

Los procesos mineros de perforación y voladura de la mina El Lucero se realizan de manera empírica, al realizar la investigación y comparación de resultados se evidencian mejoras en cuanto a rendimientos y consumo especifico de explosivos.

Respecto al tema de investigación Jáuregui (2009) expone los beneficios de la optimización de los procesos de perforación y voladura en beneficio de la reducción de costos operativos [1], Ayamamani (2016) considera fundamental la introducción de tecnología que permita mitigar al máximo los errores en las operaciones de perforación y voladura ya que esto genera sobre costos en la producción [2]. El diseño metodológico se estructura a partir del planteamiento del problema en seis momentos [3], en el primero está determinado la elaboración y consolidación del marco de referencia, posteriormente se determina la metodología con la que se aborda la problemática planteada definiendo en esta fase una investigación cuantitativa, con un enfoque experimental, realizándose el sistema de perforación y diseño de voladura; posteriormente se experimenta y se miden las variables avance, fragmentación de roca[4]. Estos datos son utilizados en la fase de análisis con un resultado 
relevante del nuevo sistema de perforación y voladura es la efectividad que se encuentra en un promedio del $96 \%$.

\section{Método}

\subsection{Macizo rocoso.}

El sistema de perforación y voladura es fundamental para identificar el tipo de conformación rocosa, permitiendo así determinar las especificaciones técnicas que se deben tener en cuenta a la hora de implementar y efectuar el diseño de un sistema de perforación y voladura adecuado. Desde esta perspectiva la caracterización del macizo rocoso es fundamental, los parámetros geotécnicos en los que se basa son: a) la resistencia al corte, b) la deformabilidad, c) la permeabilidad y d) el estado original de esfuerzos, tanto para macizos en rocas duras como en rocas blandas [5].

Es importante, además, tener en cuenta medidas tales como el Índice de resistencia geológica (GSI), la clasificación geomecánica (RMR) y el porcentaje (RQD) ya que estas medicines afectan directamente el cálculo de la contante de la roca.

\subsection{Sistemas de perforación.}

Dentro de un sistema de explotación minera la perforación es fundamental ya que la realización de esta depende la voladura, las actividades de perforación se pueden dividir en dos fases, en la primera se agrupan las acciones relacionadas a la preparación para el acceso al yacimiento, y en la segunda la preparación de los cuarteles [6]. Los sistemas de perforación se pueden clasificar en perforación a percusión y perforación a rotación [6].

\subsubsection{Sistema de perforación a rotación.}

Los sistemas de perforación a rotación son aquellos en los que la fragmentación de la roca se produce principalmente por compresión, corte o por la acción combinada de ambos [7].

\subsubsection{Sistema de perforación a percusión.}

Esta denominación engloba todas aquellas formas de perforación en las que la fragmentación de la roca, se produce básicamente por impacto, el rendimiento de este método de perforación está directamente relacionado a la potencia de percusión [7]. Dentro de los sistemas de perforación por percusión se encuentra la 1) perforación neumática y 2) la perforación hidráulica, para la determinación de un sistema óptimo de perforación se tiene que tener en cuenta la funcionalidad de cada uno de los métodos y sus costos.

\subsection{Sistemas de voladura.}

El diseño de voladura hace referencia a la disposición de un grupo de barrenos, cargados con una cantidad determina de explosivos, la activación de estos se hace siguiendo una secuencia que permita lograr resultados de fragmentación y desplazamiento ajustados a un plan operativo [8].
El diseño técnico de la malla de perforación y voladura se basa en la utilización de fórmulas matemáticas propuestas por Langefors y Kihlstrom [8] que tienen en cuenta particularidades del macizo rocoso, los explosivos y el diseño de la malla de perforación.

La mina El Lucero es una explotación subterránea de carbón, para lo cual los sistemas de iniciación y los explosivos a utilizar son permisibles. Este tipo de explosivos y detonadores tienen unas características especiales que no producen la detonación no deseada de la acumulación de gases nocivos mezclados con polvo fino de carbón o gas grisú.

\subsection{Experimentación y registro de mediciones.}

Teniendo en cuenta el objeto de estudio definido como el análisis para la optimización y aumento de la eficacia de los procesos de perforación y voladura en la mina subterránea de carbón el Lucero ubicada en la vereda de Peñas de Cajón del municipio de Sutatausa Cundinamarca, se realizaron los cálculos y el diseño de la malla de voladura.

Para realizar la caracterización de la roca, de acuerdo a los parámetros establecidos por Bienaiwsky, (1989) se infiere que el índice de calidad de la roca (RQD) es bueno, al igual, la clasificación geomecánica RMR con un puntaje de 62 lo ubica en la categoría II. Por otra parte; la resistencia a la comprensión se ubica dentro del parámetro bajo, con respecto al índice de esfuerzo geológico (GSI) según la clasificación expuesta por Hoek (1985), afirmando que la roca se encuentra muy bloquizada [9].

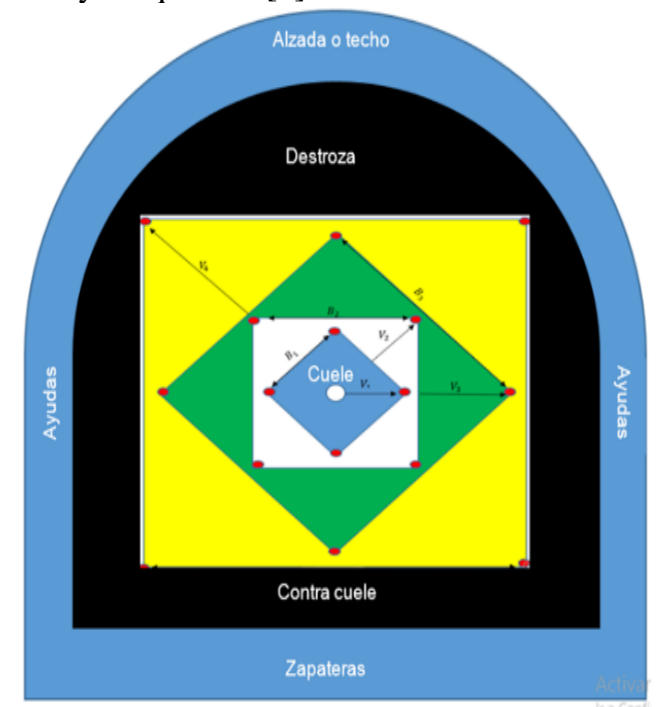

Figura 1. Denominación de taladros malla de perforación. Fuente: [6]

La dureza de la roca y las características del macizo rocoso de la mina el Lucero según los parámetros analizados es intermedia. Teniendo en cuenta estos parámetros se diseña la nueva malla de perforación y voladura de la mina el Lucero, se utiliza el método planteado por Langefors y Kihlstrom, 
este diseño se divide en: a) cuele, b) ayudas, c) zapatera y d) alza o techo, en la Fig. 1 se especifica la ubicación de cada una de estas secciones.El túnel principal tiene un área total de $5 m^{2}$, de acuerdo a las características del macizo rocoso se utilizan 22 taladros o barrenos con un consumo especifico de 0.472 kilogramos de explosivo por metro cubico de roca volada.

A partir de los requerimientos técnicos y los cálculos realizados empleado las fórmulas de Langefors y Kihlstrom se presentan el diseño técnico y las especificaciones de la nueva malla de perforación y voladura, para el desarrollo del diseño, se empleó el siguiente procedimiento y Ec (1-19).

Área del túnel.

$$
\begin{gathered}
\mathrm{A}_{1}=\mathrm{l} \times \mathrm{l} \\
A_{2}=\pi \times r^{2} / 4
\end{gathered}
$$

Numero de taladrados.

$$
N_{\text {taladros }}=\left(\frac{p}{d t}\right)+(C * S) \text {. }
$$

Avance de perforación (H).

$H=0.15+(34.1 * \emptyset)-\left(39.4 * \emptyset^{2}\right)$

Avance real $\left(H_{\text {real }}\right)$

$$
H_{\text {real }}=0.95 * H
$$

Calculo densidad del explosivo $\left(L_{\max }\right)$.

$$
L_{\max }=\frac{\pi d^{2}}{4} * D
$$

Desviación de perforación.

$Y=0.0013 H^{2}+0.0078 H+0.0014$

Desviación máxima.

$$
\Delta_{\max }=Y * H_{\text {real }}
$$

Rango teórico de consumo específico (q)

$$
\begin{aligned}
& q_{\min }=\frac{10}{A}+0.6 \\
& q_{\max }=\frac{14}{A}+0.8
\end{aligned}
$$

Consumo específico (C.E)

$$
C . E=\frac{0.56 \operatorname{Pr} \tan \left(\frac{G S I+15}{2}\right)}{\frac{\sqrt[3]{115-R Q D}}{3.3}}
$$

Calculo constante de roca " $C$ " sueco por formula.

$$
c=0.8784(C . E .)+0.052
$$

Para las secciones geométricas del cuele y las demás secciones de la malla de perforación se emplean las siguientes formulas:

Piedra optima de la primera sección $\left(V_{1}^{\prime}\right)$.

$$
V_{1}^{\prime}=V_{1}-\Delta_{\max }
$$

Valor del espacio primera sección $\left(B_{1}\right)$.

$$
B_{1}=V^{\prime}{ }_{1} \sqrt{2}
$$

Concentración lineal de explosivo $\left(L_{1}\right)$ : cantidad de carga explosiva por metro de taladro.

$$
\begin{array}{r}
L_{1}=55 * d *\left(\frac{V_{1}}{\emptyset}\right)^{1.5} *\left(V_{1}-\frac{\emptyset}{2}\right) \\
*\left(\frac{c}{0.4}\right)\left(\frac{1}{s}\right)
\end{array}
$$

Una vez estimado $L_{1}$, se procede a calcular la carga de explosivo $(Q)$ para la primera sección

$$
Q_{1}=L_{1} * H_{\text {real }}
$$

Secciones geométricas 2,3 y 4 del cuele.

$$
0.5 B_{(x-1)} \leq V_{x} \leq 2 B_{(x-1)}
$$

Espaciado de secciones 2, 3 y 4 se usa la siguiente formula.

$$
B_{x}=\left(V_{x}^{\prime}+\frac{B_{(x-1)}}{2}\right) * \sqrt{2}
$$

En la Fig. 2 se aprecian la distribución de los barrenos producto de los cálculos realizados a partir de las formulas anteriormente expuestas.

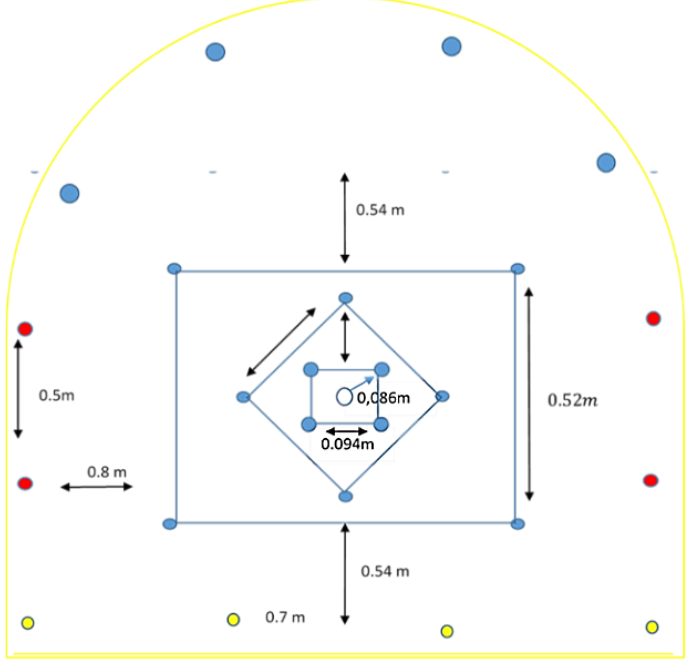

Figura 2. Malla de perforación según requerimientos técnicos. Fuente: Autores

En el trabajo de campo se analizaron los sistemas utilizados de manera empírica y en base a estos se realizó el diseño del sistema de perforación y voladura teniendo en cuenta cálculos matemáticos.

\section{Resultados}

Dentro del proceso de voladura la perforación es fundamental, es la actividad más importante ya que los errores cometidos en este procedimiento afectan de forma directa los resultados de la voladura. Desde esta perspectiva el procedimiento de perforación se realizó con rigurosidad y buscando evitar al máximo errores.

\subsection{Resultados proceso de perforación y voladura}

Para el diseño técnico de la malla de perforación se consideró la constante de roca (c) de 0.47 , el diámetro del barreno de 
alivio de $0.0508 \mathrm{~m}$, diámetro de perforación de producción (Ø) igual a $0.0381 \mathrm{~m}$ y la potencia relativa del explosivo de 1.58; estos factores determinaron el Burden y espaciamiento a utilizar y el factor de carga de cada barreno.

\subsection{Efectividad en el procedimiento}

Con los cálculos obtenidos se realizó la perforación y cargue de la malla de voladura. Se realizaron tres voladuras siguiendo los resultados obtenidos.

En la Tab. 1 se observa el avance que se obtuvo producto de las correspondientes voladuras, a partir de estos valores se determinó que el avance está en un promedio de 1,27 m.

Tabla 1. Mediciones de avance por voladura.

\begin{tabular}{cc}
\hline Voladura & Avance $(\mathrm{m})$ \\
\hline 1 & $1,28 \mathrm{~m}$ \\
\hline 2 & $1,30 \mathrm{~m}$ \\
\hline 3 & $1,25 \mathrm{~m}$ \\
\hline Promedio & $1.27 \mathrm{~m}$ \\
\hline Efectividad & $96 \%$ \\
\hline \multicolumn{2}{c}{ Fuente: Autores }
\end{tabular}

Al comparar con los resultados obtenidos de la malla que se utiliza en la mina y el nuevo diseño, se evidencio que la malla de perforación y voladura siguiendo requerimientos técnicos permite un avance de 0.25 metros más por tiro, así pues, en 4 tiros se está ganando 1 metro de avance utilizando la misma cantidad de explosivo por barreno.

\subsubsection{Efectividad en el procedimiento en los barrenos de cuele}

Anteriormente el diseño de la malla de voladura y el proceso de perforación, se realizaba con barrenos paralelos al barreno de alivio, con lo cual se generaba un promedio máximo de avance de voladora de $77.3 \%$, Tab. 2 .

Con el nuevo esquema de perforación, se implementó un diseño de la malla de voladura con cuele en cuña, al cual se le dio un incremento del $10 \%$ a la longitud de perforación $(1,40 \mathrm{~m})$, con este diseño se obtuvo resultados de avance mínimo de 96\%, mejorando rendimiento en el avance, en la granulometría del material y en el rendimiento al momento de realizar el descargue, Tab.3.

Tabla 2. Avance con perforación tradicional de la mina El Lucero

\begin{tabular}{cc}
\hline VOLADURA & AVANCE EN (M) \\
\hline 1 & $1,00 \mathrm{~m}$ \\
\hline 2 & $1,15 \mathrm{~m}$ \\
\hline 3 & $0,91 \mathrm{~m}$ \\
\hline PROMEDIO & $1,02 \mathrm{~m}$ \\
\hline EFECTIVIDAD & $77,35 \%$ \\
\hline
\end{tabular}

Tabla 3. Mediciones de avance de cuele en la nueva malla de perforación

\begin{tabular}{cc}
\hline VOLADURA & AVANCE EN (M) \\
\hline 1 & $1,40 \mathrm{~m}$ \\
\hline 2 & $1,43 \mathrm{~m}$ \\
\hline 3 & $1,39 \mathrm{~m}$ \\
\hline PROMEDIO & $1,4 \mathrm{~m}$ \\
\hline EFECTIVIDAD & $96 \%$ \\
\hline
\end{tabular}

3.3. Efectividad en el procedimiento con la utilización de barrenos ayudantes

En la mina El Lucero al realizar la malla de perforación, no se tenía en cuenta la utilización de barrenos ayudantes, por lo cual el resultado de la voladura era deficiente debido al mayor espaciamiento entre los barrenos (laterales, techeros y zapateras), esto influía en la granulometría (sobre-tamaños), rendimiento al momento de realizar el descargue y aumentos de costos.

Con el nuevo diseño de voladura se implementa la perforación de barrenos ayudantes con un espaciamiento de $0,60 \mathrm{~m}$ en relación a los barrenos de cuele, generando un resultado eficiente en el avance de la voladura, mejor granulometría y facilidad de descargue, disminuyendo costos de producción.

\section{Discusión de resultados}

De los aspectos evaluados con la implementación de la nueva malla de voladura en el avance del túnel de producción, fueron la sobre excavación y el debilitamiento del macizo rocoso.

Con respecto a la sobre excavación no se observó, la voladura realizo un corte limpio y se conservaron las dimensiones iniciales del túnel. Las paredes y techo mantienen una forma uniforme, con presencia de una cantidad pequeña de roca suelta y un fracturamiento mínimo en el macizo rocoso disminuyendo la cantidad de diaclasas. En ese orden de ideas la voladura se puede catalogar como buena y/o eficiente ya que cumple a cabalidad el objetivo para el cual fue diseñada.

Un aspecto importante en el diseño de las mallas de perforación es el concerniente a las variables que inciden en cada uno de los procesos, las decisiones que se tomaron se fundamentan en la eficiencia y la reducción de costos, aspectos vitales dentro de la producción minera.

El diseño se fundamentó en procedimientos técnicos y cálculos matemáticos para identificar la opción que más se adecue a los requerimientos de la mina el Lucero. Se realizaron tres voladuras con el procedimiento que trabajaba la mina, tres voladuras con el procedimiento propuesto y los resultados se compararon con cada uno de los ensayos observando una mejora en los resultados.

El diseño de las mallas de perforación se realizó a partir de los cálculos encontrados al realizar la indagación 
bibliográfica de varios autores, los cuales se utilizaron para mejorar las necesidades específicas de la explotación en la mina El Lucero.

En cuanto a datos de roca extraída por voladura fue de 6.35 $\mathrm{m}^{3}$ de roca, teniendo en cuenta la densidad de la roca se está hablando de 16.20 toneladas de material fragmentado por tiro, con un consumo de $2,54 \mathrm{~kg}$ de explosivo por $\mathrm{m}^{3}$, esto fue un incremento gracias al aumento en la longitud de perforación y el mejor aprovechamiento de la energía brindada por el explosivo.

Cabe resaltar que en los ensayos realizados no fue necesario realizar mayores trabajos de segmentación del material volado y un operario despejo el área volada en 4 horas. Con respecto al costo del explosivo utilizado en la voladura es de \$ 257038 pesos, teniendo en cuenta el este valor es indispensable la efectividad en el proceso de voladura. Esto significa que mejoro el rendimiento y se disminuyó el consumo de explosivo.

\section{Conclusiones}

Los procesos de perforación y voladura deben ser diseñados atendiendo a las características geológicas y geomecánicas del macizo rocoso lo que permite la consecución de resultados efectivos, el manejo adecuado de las variables que inciden en es estos resultados permite mantener los costos de producción dentro de los mínimos y que las actividades que se ejecutan cumplan a cabalidad el fin para el cual fueron planteadas. La distribución adecuada de los barrenos y sus respectivas cargas permite que no se generen discontinuidades en los trabajos de perforación y voladura, así mismo las labores de extracción del material arrancado se realiza de forma rápida. La elección del explosivo al igual que el método de detonación determinan la calidad en los procedimientos de voladura, cabe aclarar que el material idóneo para realizar voladuras en minería subterránea de carbón es el explosivo permisible junto con la utilización de detonadores permisibles. La secuencia de encendido deberá garantizar la disposición de una cara libre para que el material volado se pueda desplazar. Las actividades de perforación y voladura se configuran en un sistema de engranaje secuencial, el adecuado desarrollo de cada uno de estos procedimientos permite que el sistema funcione y cumpla las metas para las cuales se diseñó.

\section{Referencias}

[1] O. A. J. Aquino, Reducción de los Costos Operativos en Mina, mediante la optimización de los Estándares de las operaciones unitarias de perforación y Voladura, Lima-Perú: Pontificia universidad de Perú, 2009.

[2] C. Ayamamani Pachari, Diseño de perforación y voladura y su incidencia en los costos unitarios en Balcón III de la corporación minera Ananea SA., Universidad Nacional del Altiplano, Puno Perú , 2016.
[3] R. Hernández Sampieri, C. Fernández Collado, P. Baptista Lucio, Metodología de la Investigación, México : McGRAWHILL Interamericana Editores, SA DE C.V, 2004.

[4] D. Duque Escobar, Macizo rocoso manual de geología para ingenieros, 2003, pp. 307-337.

[5] M. Estruch Serra, A. Tapia Gómez, Topografía subterránea para minería y obras, Barcelona: Ediciones UPC, 2010.

[6] J. Castilla Gómez, J. Herrera Herbert, Perforación y voladura de rocas en minería, Madrid: Universidad Politécnica de Madrid. Departamento de Explotación de Recursos Minerales y Obras Subterráneas. Laboratorio de Tecnologías Mineras, 2013.

[7] López Jimeno. Manual de perforación y voladura de roca. Instituto Geominero de España.

[8] EXSA, Manual Práctico de Voladura, Perú: Centro Tecnológico de Voladura EXSA., 2013.

[9] C. A. Ortega Ramosl, A. F. Jaramillo Gil, J. M. Molina Escobar, "Modificación de las mallas de perforación de voladuras a partir del índice de esfuerzo geológico (GSI), caso mina "La Maruja", Colombia”, Boletín de Ciencias de la Tierra, No. 40, julio, 32-38, 2016. 